\title{
A multi-institutional analysis of sternoclavicular joint coverage following osteomyelitis
}

\author{
Sammy Othman ${ }^{1}$, Omar Elfanagely ${ }^{1}$, Saïd C. Azoury ${ }^{1}$, Geoffrey M. Kozak², Jessica Cunning ${ }^{1}$, \\ Arturo J. Rios-Diaz ${ }^{1}$, Prashanth Palvannan ${ }^{2}$, Patrick Greaney ${ }^{2}$, Matthew P. Jenkins ${ }^{2}$, Doraid Jarrar', \\ Stephen J. Kovach ${ }^{1}$, John P. Fischer ${ }^{1}$ \\ ${ }^{1}$ Division of Plastic Surgery, Department of Surgery, University of Pennsylvania, Philadelphia, PA; ${ }^{2}$ Division of Plastic Surgery, Department of \\ Surgery, Thomas Jefferson University, Philadelphia, PA; ${ }^{3}$ Division of Thoracic Surgery, Department of Surgery, University of Pennsylvania, \\ Philadelphia, PA, USA
}

Background Sternoclavicular joint (SCJ) osteomyelitis is a rare pathology requiring urgent intervention. Several operative approaches have been described with conflicting reports. Here, we present a multi-institutional study utilizing multiple surgical pathways for SCJ reconstruction. Methods A multi-institutional retrospective cohort study was conducted to identify patients who underwent surgical repair for sternoclavicular osteomyelitis between 2008 and 2019. Patients were stratified according to reconstruction approach: single-stage reconstruction with advancement flap and delayed-reconstruction with flap following initial debridement. Demographics, operative approach, type of reconstruction, and postoperative outcomes were analyzed. Results Thirty-two patients were identified. Mean patient age was $56.2 \pm 13.8$ years and $68.8 \%$ were male. The average body mass index (BMI) was $30.0 \pm 8.8 \mathrm{~kg} / \mathrm{m}^{2}$. The most common infection etiologies were intravenous drug use and bacteremia (both 25\%). Fourteen patients (43.8\%) underwent one-stage reconstruction and 18 (56.2\%) underwent delayed twostaged reconstruction. Both single and delayed-stage groups had comparable rates of reinfection (7.1\% vs. $11.1 \%$, respectively), surgical site complications (21.4\% vs. $27.8 \%)$, readmissions (7.1\% vs. 16.6\%), and reoperations (7.1\% vs. 5.6\%; all P>0.05). The single-stage reconstruction group had a significantly lower BMI $\left(26.2 \pm 5.7 \mathrm{~kg} / \mathrm{m}^{2}\right.$ vs. $\left.32.9 \pm 9.1 \mathrm{~kg} / \mathrm{m}^{2} ; \mathrm{P}<0.05\right)$ and trended towards shorter hospital length of stay (11.3 days vs. 17.9 days; $P=0.01$ ).

Conclusions Both single and delayed-stage approaches are appropriate methods with comparable outcomes for reconstruction for SCJ osteomyelitis. When clinically indicated, a singlestage reconstruction approach may be preferable in order to avoid a second operation as associated with the delayed phase, and possibly shortening total hospital length of stay.

Keywords Osteomyelitis / Surgery, plastic / Reconstructive surgery / Clavicle
Correspondence: Sammy Othman Division of Plastic Surgery, University of Pennsylvania, Perelman Center for Advanced Medicine, 3400 Civic Center Blvd, Philadelphia, PA 19104, USA

Tel: +1-732-910-5021

Fax: +1-215-349-8312

E-mail:sothman13@gmail.com

\section{INTRODUCTION}

Infection of the sternoclavicular joint (SCJ) can present with the need for urgent intervention due to a propensity for insidious manifestation and spread [1]. Symptoms may include fever, marked pain and tenderness, erythema, and decreased mobility, 
but are often unspecific [2-4]. Risk factors include intravenous drug use (IVDU), but reports of indwelling catheterization, traumatic etiology, exposed wound beds, immunosuppression, diabetes mellitus (DM), chronic hepatic and renal disease, and autoimmune disease have also been reported [1,5-8]. Severe infection can further spread as osteomyelitis, which requires urgent action due to the SCJ's anatomical proximity to the great vessels. Left untreated, patients with infection of the SCJ may suffer from large surface-area resection, mediastinitis, sepsis, and possibly death, with a mediastinitis rate of $13 \%$ and a mortality rate of up to $4 \%$, even in cases where patients are treated $[1,3,9]$.

Treatment options have ranged from nonoperative management to multiple operative strategies, as well as several subsets of combinations of the two therein. However, the most effective approaches are not well-described. Medical therapy with antibiotics has resulted in undesirable morbidity rates [1]. Surgical approaches include percutaneous incision and drainage (I\&D), debridement, simple resection with or without negative pressure wound therapy (NPWT) and delayed wound healing, onestage resection and reconstruction, and/or a hybrid combination of techniques [6,10-12]. There has been increased focus on addressing the dead-space and heightened risk of vessel exposure created by SCJ resection, and this can be addressed given the adjacent soft-tissue $[13,14]$. Traditionally, the pectoralis major muscle has been used for these purposes, though there are reports of the latissimus dorsi flap advancement as well [14].

A challenging aspect of delineating the efficacy of each treatment modality is the relative rarity and low incidence of sternoclavicular (SC) osteomyelitis, with scarce data on management generally limited to small series studies $[8,9,11-15]$. For these reasons, the more appropriate management strategies have been debated over several decades and are consistently evolving particularly pertaining to technique and timing of reconstruction. To our knowledge, we present the first multi-center and multiinstitutional review of the multiple surgical approaches to the management of SCJ infection.

\section{METHODS}

\section{Study population}

Following institutional review board approval (IRB No. 834502), a multi-institutional review was performed to identify patients who had undergone SCJ reconstruction following sternoclavicular (SC) osteomyelitis from January 2008 to October 2019. Patients were included if they were $\geq 18$ years of age and had undergone surgical SCJ reconstruction following resection of the SCJ due to osteomyelitis. All etiologies of osteomyelitis were considered. Patients with SCJ resection due to noninfectious etiology, such as oncological indications, were excluded.

\section{Data source and covariates}

Demographic, clinical and operative data were extracted from the electronic medical record. Demographics data collected included age, sex, ethnicity, and body mass index (BMI), comorbidities such as hypertension, DM, smoking status, obesity (defined as BMI $\geq 30 \mathrm{~kg} / \mathrm{m}^{2}$ ), chronic liver disease, defined as presence of cirrhosis or chronic hepatitis, chronic kidney disease, and immunosuppressed status, defined as actively taking known immunosuppressive medications and/or the presence of an immunosuppressive condition such as cancer or HIV (human immunodeficiency virus). Infectious disease details were also extracted and these included suspected source of infection and microbiology data from cultures. All patients were diagnosed based upon clinical exam, with the aid of computed tomography and/or magnetic resonance imaging preoperatively, and culture/biopsy sample. Patients were stratified according to operative approach (one-stage vs. delayed reconstruction). Onestage reconstruction was defined as SC resection followed by flap coverage during the same operation without prior debridement. Delayed reconstruction was defined as flap coverage in a subsequent operation after one or multiple operations for SCJ debridement.

\section{Outcomes}

Postoperative data was evaluated, which included surgical site complications, reoperations, readmissions hospital length of stay (LOS), and date of last follow-up. The primary outcome was infection resolution following the index reconstructive procedure, defined as a lack of significant findings on clinical exam and objective laboratory and imaging measures to the date of last follow-up. Secondary outcomes included surgical site complications, need for reoperation, readmission, and hospital LOS.

\section{Operative strategy}

All patients underwent initial debridement, and/or SJC resection during the index procedure. The feasibility of one-stage flap closure was largely dictated by the extent of infection, purulence, and effectiveness of primary resection and/or debridement of the SCJ, clavicle, plus or minus the first rib, down to healthy bone, dependent upon intraoperative findings. Cultures were then obtained from bone and soft-tissue. If the infection was believed to be largely cleared, a pectoralis major advancement flap was utilized for joint reconstruction. When further resection and/or debridement was mandated, reconstruction with an advancement flap was delayed to a later date following satisfactory clearance of frank infection (Fig. 1). In the intermediate period, 
Fig. 1. Imaging confirmed osteomyelitis of the sternoclavicular joint

A young woman presented with imaging-confirmed osteomyelitis of the sternoclavicular joint. (A) Intraoperative dead space following resection of infected sternoclavicular joint. (B) Infected specimen following resection. (C) Inset of the mobilized pectoralis major flap within the sternoclavicular dead space following medial joint resection. (D) Immediate postoperative incisional closure.
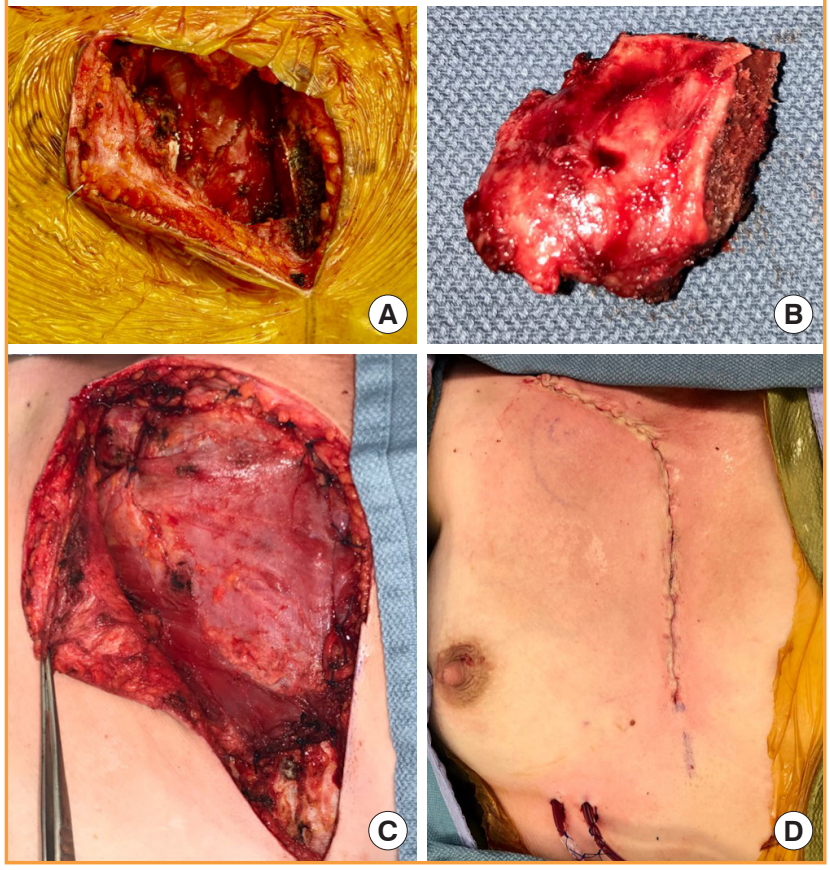

NPWT was used, given its known advantages in wound granulation and potential for infection clearance.

\section{Statistical analysis}

Descriptive statistics were used to categorize incidence of variables of interest. Categorical variables were analyzed using chisquare testing and Fisher exact test, where appropriate, while continuous variables were compared using Mann-Whitney $U$ testing. Statistical significance was set at $\mathrm{P} \leq 0.05$. Statistical calculations and analyses were performed using IBM SPSS version 25 (IBM Corp., Armonk, NY, USA).

\section{RESULTS}

A total of 32 patients who underwent SCJ reconstruction following SC osteomyelitis were deemed appropriate for inclusion and analysis. All patients in this study were diagnosed with definitive or likely osteomyelitis preoperatively, which was later confirmed during surgical biopsy. Imaging modalities diagnosed 11 (34.4\%) patients with definitive osteomyelitis and 14 (43.8\%) with possible osteomyelitis, while the remaining seven (21.9\%) were nondiagnostic/inconclusive. Patients were aged $56.2 \pm 13.8$ years-
Table 1. Patient demographics and comorbidities

\begin{tabular}{|lcccc|}
\hline Characteristic & $\begin{array}{c}\text { All patients } \\
(\mathbf{n}=\mathbf{3 2})\end{array}$ & $\begin{array}{c}\text { One-stage } \\
(\mathbf{n}=14)\end{array}$ & $\begin{array}{c}\text { Delayed } \\
(\mathbf{n}=18)\end{array}$ & P-value \\
\hline Age (yr) & $56.2 \pm 13.8$ & $56.1 \pm 14.9$ & $56.2 \pm 13.4$ & 0.954 \\
Male sex & $22(68.8)$ & $9(64.3)$ & $13(72.2)$ & 0.631 \\
Ethnicity & & & & 0.424 \\
$\quad$ Caucasian & $21(65.6)$ & $8(57.1)$ & $13(72.2)$ & \\
$\quad$ African American & $10(31.3)$ & $5(35.7)$ & $5(27.8)$ & \\
$\quad$ Hispanic & $1(3.1)$ & $1(7.1)$ & 0 & \\
BMI (kg/m $\left.{ }^{2}\right)$ & $30.0 \pm 8.8$ & $26.2 \pm 5.7$ & $32.9 \pm 9.1$ & $0.009^{\mathrm{a}}$ \\
Obesity & $14(43.8)$ & $4(28.6)$ & $10(71.4)$ & 0.165 \\
HTN & $20(62.5)$ & $9(64.3)$ & $11(61.1)$ & 0.854 \\
DM & $14(43.8)$ & $7(50)$ & $7(38.9)$ & 0.530 \\
Smoking status & & & & 0.310 \\
$\quad$ Never & $14(43.8)$ & $4(28.6)$ & $10(71.4)$ & \\
Former & $7(21.9)$ & $4(28.6)$ & $3(16.7)$ & \\
$\quad$ Current & $11(34.4)$ & $6(42.9)$ & $5(27.8)$ & \\
Immunocompromised & $6(18.8)$ & $2(14.3)$ & $4(22.2)$ & 0.672 \\
Chronic kidney disease & $9(28.1)$ & $4(28.6)$ & $5(27.8)$ & 1.000 \\
Chronic liver disease & $4(12.5)$ & $1(7.1)$ & $3(16.7)$ & 0.613 \\
\hline
\end{tabular}

Values are presented as mean $\pm \mathrm{SD}$ or number $(\%)$.

BMI, body mass index; HTN, hypertension; DM, diabetes mellitus.

aStatistically significant, $\mathrm{P}<0.05$.

old at the time of surgery, $68.8 \%$ were male, $43.8 \%$ were obese, $62.5 \%$ hypertensive, $43.8 \%$ diabetic, $18.8 \%$ were immunosuppressed, $28.1 \%$ had chronic kidney disease, and $12.5 \%$ had chronic liver disease. Patients of $21.9 \%$ were former smokers., while $34.4 \%$ identified as current smokers at the time of the procedure. BMI was significantly higher in delayed group (32.9 \pm $\left.9.1 \mathrm{~kg} / \mathrm{m}^{2}\right)$, compared to the one-stage group $\left(26.2 \pm 5.7 \mathrm{~kg} / \mathrm{m}^{2}\right)$ $(\mathrm{P}=0.009)$. No other characteristics differed significantly between groups (Table 1 ).

The majority of patients presented with right-sided osteomyelitis of the SCJ (65.6\%). The most common pre-disposing etiologies for infection were IVDU (25\%) and distant bacteremia (25\%). The least common etiology was a central venous catheter (3.1\%). Other etiologies included local abscess spread, trauma, and cardiac based infection (all 9.4\%, respectively). Chronic immunosuppressed state was believed to serve as the etiology for $6.3 \%$ of patients, who were both taking immunosuppressive medications. Source of infection was unknown in $12.5 \%$ of patients. Etiologies were similar across approaches (Table 2). Fourteen patients (43.8\%) underwent one-staged resection and flap-reconstruction. The remaining 18 (56.2\%) underwent a delayed approach with primary attempt at infection control followed by eventual definitive flap reconstruction. The median number of operative procedures for this group prior to definitive flap closure was 1.5. With regards to the delayed group, prior to final flap reconstruction, $38.9 \%$ (7/18) underwent SCJ resection, 33\% (6/18) underwent both I\&D and resection, and 


\section{Table 2. Infective characteristics}

\begin{tabular}{|lcccc|}
\hline Characteristic & $\begin{array}{c}\text { All patients } \\
(\mathbf{n}=\mathbf{3 2})\end{array}$ & $\begin{array}{c}\text { One-stage } \\
(\mathbf{n}=14)\end{array}$ & $\begin{array}{c}\text { Delayed } \\
(\mathbf{n}=18)\end{array}$ & P-value \\
\hline Laterality & & & & 0.061 \\
$\quad$ Right SCJ & $21(65.6)$ & $12(85.7)$ & $9(50)$ & \\
Etiology & & & & 0.881 \\
IVDU & $8(25.0)$ & $3(21.4)$ & $5(27.8)$ & \\
Catheter-related & $1(3.1)$ & 0 & $1(5.6)$ & \\
Local spread & $3(9.4)$ & $2(14.3)$ & $1(5.6)$ & \\
Bacteremia & $8(25.0)$ & $4(28.6)$ & $4(22.2)$ & \\
Cardiac infection & $3(9.4)$ & $1(7.1)$ & $2(11.1)$ & \\
Trauma & $3(9.4)$ & $2(14.3)$ & $1(5.6)$ & \\
Immunosuppression & $2(6.3)$ & $1(7.1)$ & $1(5.6)$ & \\
Unknown & $4(12.5)$ & $1(7.1)$ & $3(16.7)$ & \\
Microbiology & & & & 0.926 \\
Staphylococcus aureus & $17(53.1)$ & $7(50.0)$ & $10(55.6)$ & \\
Staphylococcus epidermidis & $2(6.3)$ & $1(7.1)$ & $1(5.6)$ & \\
Streptococcus species & $2(6.3)$ & $1(7.1)$ & $1(5.6)$ & \\
Gram-negative & $3(9.4)$ & $2(14.3)$ & $1(5.6)$ & \\
Anaerobe & $4(12.5)$ & $2(14.3)$ & $2(11.1)$ & \\
Unknown & $4(12.5)$ & $1(7.1)$ & $3(16.7)$ & \\
\hline
\end{tabular}

Values are presented as number (\%).

SCJ, sternoclavicular joint; IVDU, intravenous drug use.

$27.7 \%(5 / 18)$ underwent I\&D/debridement with no subsequent resection prior to flap-reconstruction. Wound vacuum assisted closure was applied to all delayed-closure patient wounds until the time of the definitive reconstructive procedure.

Staphylococcus aureus was the most common organism identified on microbiological culture (53\% of all cases), while Staphylococcus epidermidis and Streptococcus species were the least common (6.3\% each, respectively). Anaerobic bacteria (12.5\%) and Gram-negative bacteria (9.4\%) composed the other known pathogens of infection, which were all similar across both groups (Table 2).

Eight total patients (25\%) experienced wound-site complications (Table 3) over a median follow-up of 4 months (range, 1-21 months). Three patients (9.4\%) had seromas postoperatively, and two $(6.3 \%)$ had hematomas. One hematoma required reoperative evacuation, with the remaining four of these fluid collections managed nonoperatively. The remaining three patients (9.4\%) with wound-site complications experienced infection recurrence. In all three cases, the SCJ was initially resected before flap coverage. The first of these patients also had his first rib resected with the initial SCJ resection, but required further debridement and second rib resection following recurrence in order to achieve resolution. The second patient, who had both his SCJ and first rib resected initially, necessitated reoperation, but was not performed due to continuous IVDU. The final patient, who only had his SCJ resected, was found to have simultaneous endocarditis and bacteremia as a result of infection,
Table 3. Postoperative outcomes

\begin{tabular}{|lcccc|}
\hline Characteristic & $\begin{array}{c}\text { All patients } \\
(\mathbf{n}=\mathbf{3 2})\end{array}$ & $\begin{array}{c}\text { One-stage } \\
(\mathbf{n}=\mathbf{1 4})\end{array}$ & $\begin{array}{c}\text { Delayed } \\
(\mathbf{n}=\mathbf{1 8})\end{array}$ & P-value \\
\hline Wound complications (any) & $8(25.0)$ & $3(21.4)$ & $5(27.8)$ & 1.00 \\
Seroma & $3(9.4)$ & $1(7.1)$ & $2(11.1)$ & \\
Hematoma & $2(6.3)$ & $1(7.1)$ & $1(5.6)$ & \\
Reinfection & $3(9.4)$ & $1(7.1)$ & $2(11.1)$ & 1.00 \\
Readmissions & $4(12.5)$ & $1(7.1)$ & $3(16.6)$ & 0.61 \\
Reoperations & $2(6.3)$ & $1(7.1)$ & $1(5.6)$ & 1.00 \\
Length of stay (day) & $15.0 \pm 13.4$ & $11.3 \pm 10.3$ & $17.9 \pm 15.4$ & 0.098 \\
\hline \multicolumn{4}{l}{ Values are presented as number (\%) or mean \pm SD. } \\
\end{tabular}

and unfortunately passed away.

In total, four patients were readmitted (12.5\%) and two underwent reoperation (6.3\%). The four readmissions involved the three infective recurrences and one hematoma, which was initially admitted due to concern for reinfection. One reoperation encompassed the aforementioned debridement and resection procedure. The second reoperation occurred in the immediate postoperative state due to an extensive hematoma. No difference in complications, readmissions, or reoperation was observed between groups $($ all $\mathrm{P}<0.05)$ (Table 3$)$.

The mean LOS for all patients was $15.0 \pm 13.4$ days. Patients in the delayed-closure group had an average greater LOS of $17.9 \pm 15.4$ days, while patients in the one-stage group experienced a stay of $11.3 \pm 10.3$ days, a difference approaching significance $(\mathrm{P}=0.098)$ (Table 3$)$.

\section{DISCUSSION}

The rare incidence of SC osteomyelitis presents challenges in identifying the proper treatment modality. DM, immunosuppressed status, catheter use, renal failure, and chronic liver disease have all been reportedly associated with SCJ infection, and may increase in incidence $[8,14,16-18]$; given these associations, SC osteomyelitis may represent a growing and more frequently encountered pathology that demands prompt diagnosis and proper management. Despite this, the data presented within this study indicates that both immediate and delayed construction are effective management approaches.

Between groups, there were no evident differences in patient characteristics with the exception of a significantly elevated BMI in the delayed-reconstruction group. Several studies have suggested that an increase in BMI is likely associated with chronic inflammation, a compromised immune system, and an increased predisposition to infection and worsened outcomes $[18,19]$. In this study, patients with an elevated BMI may have been found to have worsened infections during operative explo- 
ration, necessitating a deferral to multiple operations in order to achieve adequate infection clearance and definitive closure. As mentioned, immunosuppression and chronic disease states are associated with SCJ infection $[8,14]$. We hypothesize that an elevated BMI may predispose a similar state and influence worsened infection in the context of SC osteomyelitis.

IVDU and bacteremia were found to have the highest incidence of infective etiology. Both can result in spontaneous seeding of the SCJ. IVDU may also cause infection due to direct inoculation through injection into or near the internal jugular veins [1]. From a microbiological perspective, S. aureus was the most commonly encountered pathogen in this study, with similar rates to previously reported figures $[1,20]$, perhaps due to its ubiquity as a skin bacterium [12].

Both treatment approaches offered similar rates of reinfection, surgical site complications, reoperations, no and readmissions. LOS was markedly increased in the delayed-stage group, likely reflective of the need for one or more additional operations. Cases of reinfection and recurrence are likely due inadequate infection clearance when undergoing surgical debridement, even when closed with a vascularized flap. We hypothesize that all cases of recurrence in our study were due to insufficient debridement and resection of residually infected bony structures, including the surrounding ribs when necessary. This highlights the need for meticulous debridement in order to mitigate the risk of recurrence.

Previous authors have presented various treatment strategies. Kachala et al. [11] and Puri et al. [15] both presented their experiences comparing immediate single-stage flap reconstruction with a different delayed approach than the one we present; Both of their groups opted for debridement and open wound carewith no subsequent flap. Their findings differed, however, in that Kachala et al. suggested no difference between approaches while Puri et al. concluded the delayed approach to provide less complications. Muesse et al. [21] presented an alternative strategy by delaying both resection and coverage following initial debridement, finding that this approach provided the least complications.

In this study, a delayed approach was utilized wherever infection control was not feasible on the index procedure. The specific strategy used was dependent upon intraoperative findings, which may have necessitated resection, I\&D, and/or debridement, rather than one specific delayed-closure pathway, in contrast to the above studies where one delayed pathway was utilized. Once adequate clearance of infection was achieved, patients underwent definitive flap closure. The authors provide an algorithmic approach to help clarify management strategies for SCJ infection. The authors use preoperative imaging and intraoperative signs of

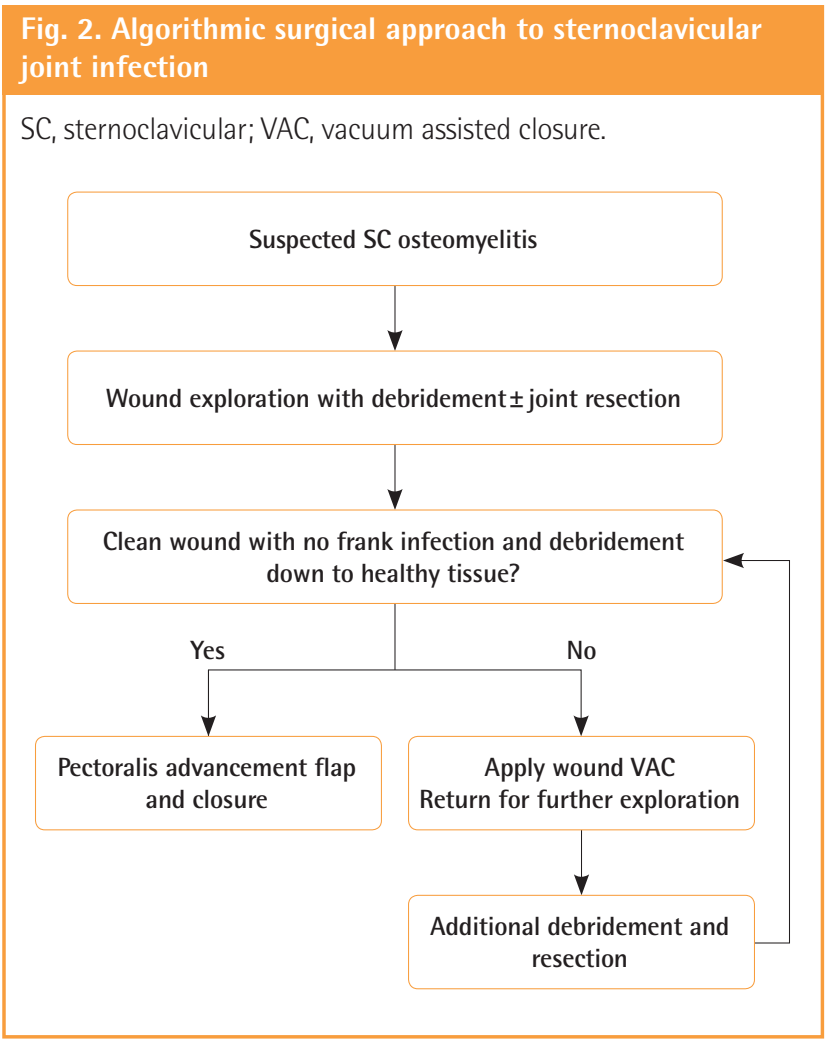

infection to determine primary versus delayed closure. This necessitates aggressive and meticulous debridement of soft-tissue and bony resection of the SCJ and surrounding ribs, if there is evidence of infective spread to these areas. If this proves inadequate on the initial operation, or if there is uncertainty about the continued presence of infection, the authors advocate for a delayed-closure pathway and return to the operating room until evidence of healthy tissue is clearly present (Fig. 2).

Previous research has indicated that unilateral medical management with intravenous antibiotic therapy is undesirable given higher reported failure rates [1]. Surgically, I\&D with an open wound managed with NPWT or left to heal by secondary intention is another reported approach, but is associated with failure rates as high as $83 \%$ and a necessity for prolonged wound care [14]. For these reasons, we generally prefer aggressive debridement, resection, and flap-based reconstruction.

One advantage of the flap is to allow filling of dead-space, a critical consideration in reducing dead-space and consequent nidus for further infection and reoperation $[22,23]$. A second purpose for flap advancement is the paramount coverage of critical vessel exposure [22,23], as brachiocephalic vessels traverse directly deep to the SCJ. All cases in this study utilized the pectoralis major flap. Although there are reports of latissimus dorsi advancement flap harvest for this purpose, the pectoralis major flap provides a less technically demanding procedure given its 
effectiveness in superomedial wound-coverage of the anterior chest [24].

An initial concern in the resection of the SCJ was a consequent compromised musculoskeletal function. Several studies have shown that functionality is not significantly inhibited by loss of joint in the setting of partial hemi-manubrial resection $[11,14$, $15]$, contrary to what was intuitively believed at the conception of this strategy. It is possible, however, that detrimental longterm effects regarding function exist but are not otherwise evident in any intermediate time-frame.

The authors are aware of several limitations of the study, including those inherent to the retrospective nature. There is potential for selection bias and confounding by indication. However, most patients' characteristics were comparable between both cohorts and this study elucidates the pathway to reconstruction and outcomes by reconstruction timing using multi-institutional data. Additionally, the average follow-up in our cohort was 4 months and therefore long-term outcomes are yet to be described. Lastly, given the rare incidence of SC osteomyelitis, the relative small sample size precludes definitive conclusions. Nonetheless, we have presented results for a relatively large cohort of patients with SCJ infection that underwent reconstruction. Similarly, specific risk factors could be identified in the future with larger studies. Further studies will do well elucidating risk factors associated with poor outcomes by surgical treatment approach in a larger population. Regardless, this study shows that single-stage and delayed-stage reconstruction approaches following debridement for SC osteomyelitis are comparably effective dependent upon the proper operative indication.

In conclusion, Both single and delayed-stage approaches are appropriate methods with comparable outcomes for reconstruction for SCJ osteomyelitis. When clinically indicated, a single-stage reconstruction approach may be preferable in order to avoid a second operation as associated with the delayed phase, and possibly shortening total hospital LOS.

\section{NOTES}

\section{Conflict of interest}

No potential conflict of interest relevant to this article was reported.

\section{Ethical approval}

The study was approved by the Institutional Review Board of the University of Pennsylvania (IRB No. 834502) and performed in accordance with the principles of the Declaration of Helsinki. Written informed consents were obtained.

\section{Patient consent}

The patients provided written informed consent for the publication and the use of their images.

\section{Author contribution}

Conceptualization: S Othman, O Elfanagely, SC Azoury, GM Kozak, P Palvannan, P Greaney, MP Jenkins, D Jarrar, SJ Kovach, JP Fischer. Data curation: S Othman, O Elfanagely, SC Azoury, GM Kozak, J Cunning, AJ Rios-Diaz, P Palvannan, JP Fischer. Formal analysis: all authors. Methodology: S Othman, O Elfanagely, SC Azoury, GM Kozak, J Cunning, AJ Rios-Diaz, P Palvannan, D Jarrar, SJ Kovach, JP Fischer. Project administration: S Othman, O Elfanagely, SC Azoury, P Greaney, MP Jenkins, D Jarrar, SJ Kovach, JP Fischer. Visualization: S Othman, O Elfanagely, J Cunning, AJ Rios-Diaz, MP Jenkins, SJ Kovach, JP Fischer. Writing - original draft: S Othman, O Elfanagely, SC Azoury, GM Kozak, AJ Rios-Diaz. Writing - review \& editing: $P$ Greaney, MP Jenkins, D Jarrar, SJ Kovach, JP Fischer.

\section{ORCID}

Sammy Othman https://orcid.org/0000-0002-8814-1048

Omar Elfanagely https://orcid.org/0000-0003-2076-0026

Saï C. Azoury https://orcid.org/0000-0002-2263-4149

Geoffrey M. Kozak https://orcid.org/0000-0001-9844-3544

Jessica Cunning https://orcid.org/0000-0001-5247-3553

Arturo J. Rios-Diaz https://orcid.org/0000-0002-0867-1920

Prashanth Palvannan https://orcid.org/0000-0003-1437-6714

Patrick Greaney https://orcid.org/0000-0002-7073-3889

Matthew P.Jenkins https://orcid.org/0000-0003-4412-5270

Doraid Jarrar ～https://orcid.org/0000-0002-1031-4463

Stephen J. Kovach https://orcid.org/0000-0002-0308-5607

John P. Fischer https://orcid.org/0000-0002-3373-1016

\section{REFERENCES}

1. Ross JJ, Shamsuddin H. Sternoclavicular septic arthritis: review of 180 cases. Medicine (Baltimore) 2004;83:139-48.

2. Moyer HR, Ghazi B, Feliciano DV. Sternoclavicular joint infection: a case report. Thorac Cardiovasc Surg 2009;57:500-1.

3. Tanaka Y, Kato H, Shirai K, et al. Sternoclavicular joint septic arthritis with chest wall abscess in a healthy adult: a case report. J Med Case Rep 2016;10:69.

4. Ely GM. Septic arthritis of the sternoclavicular joint and osteomyelitis of the proximal clavicle caused by prevotella melaninogenicus: a case with several features delaying diagnosis. J Clin Rheumatol 1999;5:354-9.

5. Dounousi E, Duni A, Xiromeriti S, et al. Acute bacterial sternoclavicular osteomyelitis in a long-term renal transplant re- 
cipient. World J Transplant 2016;6:442-6.

6. Bar-Natan M, Salai M, Sidi Y, et al. Sternoclavicular infectious arthritis in previously healthy adults. Semin Arthritis Rheum 2002;32:189-95.

7. Hunter D, Moran JF, Venezio FR. Osteomyelitis of the clavicle after Swan-Ganz catheterization. Arch Intern Med 1983; 143:153-4.

8. Abu Arab W, Khadragui I, Echave V, et al. Surgical management of sternoclavicular joint infection. Eur J Cardiothorac Surg 2011;40:630-4.

9. Murga A, Copeland H, Hargrove R, et al. Treatment for sternoclavicular joint infections: a multi-institutional study. J Thorac Dis 2017;9:1503-8.

10. Tickell KD, Banim R, Kustos I. Salmonella sternoclavicular osteomyelitis in a patient with Crohn's disease. BMJ Case Rep 2013;2013:bcr2012007809.

11. Kachala SS, D’Souza DM, Teixeira-Johnson L, et al. Surgical management of sternoclavicular joint infections. Ann Thorac Surg 2016;101:2155-60.

12. Carlos GN, Kesler KA, Coleman JJ, et al. Aggressive surgical management of sternoclavicular joint infections. J Thorac Cardiovasc Surg 1997;113:242-7.

13. Opoku-Agyeman J, Matera D, Simone J. Surgical configurations of the pectoralis major flap for reconstruction of sternoclavicular defects: a systematic review and new classification of described techniques. BMC Surg 2019;19:136.

14. Song HK, Guy TS, Kaiser LR, et al. Current presentation and optimal surgical management of sternoclavicular joint infections. Ann Thorac Surg 2002;73:427-31.

15. Puri V, Meyers BF, Kreisel D, et al. Sternoclavicular joint in- fection: a comparison of two surgical approaches. Ann Thorac Surg 2011;91:257-61.

16. Rowley WR, Bezold C, Arikan Y, et al. Diabetes 2030: insights from yesterday, today, and future trends. Popul Health Manag 2017;20:6-12.

17. Harpaz R, Dahl RM, Dooling KL. Prevalence of immunosuppression among US adults, 2013. JAMA 2016;316:25478.

18. Rodriguez-Hernandez H, Simental-Mendia LE, RodriguezRamirez G, et al. Obesity and inflammation: epidemiology, risk factors, and markers of inflammation. Int $\mathrm{J}$ Endocrinol 2013;2013:678159.

19. Dobner J, Kaser S. Body mass index and the risk of infection-from underweight to obesity. Clin Microbiol Infect 2018;24:24-8.

20. Nusselt T, Klinger HM, Freche S, et al. Surgical management of sternoclavicular septic arthritis. Arch Orthop Trauma Surg 2011;131:319-23.

21. Muesse JL, Blackmon SH, Ellsworth WA 4th, et al. Treatment of sternoclavicular joint osteomyelitis with debridement and delayed resection with muscle flap coverage improves outcomes. Surg Res Pract 2014;2014:747315.

22. Burkhart HM, Deschamps C, Allen MS, et al. Surgical management of sternoclavicular joint infections. J Thorac Cardiovasc Surg 2003; 125:945-9.

23. Pairolero PC, Arnold PG, Harris JB. Long-term results of pectoralis major muscle transposition for infected sternotomy wounds. Ann Surg 1991;213:583-9.

24. Momeni A, Kovach SJ. Important considerations in chest wall reconstruction. J Surg Oncol 2016;113:913-22. 